\title{
The Polymorphisms of Epidermal Growth Factor-driven Signaling and Cancer Pathogenesis
}

\author{
Monica Dugăeșescu1 ${ }^{1}$ Florentina Mușat ${ }^{2}$, and Octavian Andronic ${ }^{2,3}$ \\ ${ }^{1}$ Fundeni Clinical Institute, Carol Davila University of Medicine and Pharmacy, Bucharest, Romania \\ ${ }^{2}$ University Emergency Hospital of Bucharest, Romania \\ ${ }^{3}$ Carol Davila University of Medicine and Pharmacy, Bucharest, Romania
}

ORCID:

Monica Dugăeșescu: http://orcid.org/0000-0002-7672-086X

Florentina Mușat: http://orcid.org/0000-0003-2575-8646

Octavian Andronic: http://orcid.org/0000-0002-9053-0018

Corresponding Author: Monica Dugăeșescu; Fundeni Clinical Institute, Carol Davila University of Medicine and Pharmacy, Bucharest, Romania. email: monicadugaesescu@yahoo.com

Received 23 April 2021 Accepted 1 June 2021

Published 30 June 2021

Production and Hosting by Knowledge E

(c) Monica Dugăeșescu et al. This article is distributed under the terms of the

Creative Commons

Attribution License, which

permits unrestricted use and redistribution provided that the original author and source are credited.

Editor-in-Chief:

Prof. Mohammad A. M. Ibnouf

\section{Abstract}

Background: Epidermal growth factor (EGF) is a stimulating protein for cell proliferation and differentiation. An amplification of its signaling pathway has been frequently reported in numerous malignant tumors. Specific polymorphisms of the genes encoding proteins involved in this cellular pathway may constitute risk factors for carcinogenesis. The aim of this study was to identify the most relevant polymorphisms of EGF and their signaling pathways and their relation to carcinogenesis.

Methods: The study included 40 full-text articles published between January 2010 and May 2020, extracted from PubMed, Scopus, Web of Science, and Science Direct databases in May 2020, using the following keywords: EGF OR epidermal growth factor AND polymorphism AND cancer OR neoplasia OR tumor.

Results: We identified relevant polymorphisms of the EGF signaling pathway that were involved in the development and progression of hepatocellular carcinoma, esophageal cancer, gastric cancer, colorectal cancer, glioma, lung cancer, breast cancer, cervical cancer, and head and neck cancer. Rs4444903 variants have been widely studied and the association with numerous tumors has been confirmed by multiple studies. Other frequently investigated polymorphisms are $-191 \mathrm{C} / \mathrm{A}$ and $-216 \mathrm{G}>\mathrm{T}$.

Conclusion: The polymorphisms of EGF signaling pathway have been widely studied in connection to various malignancies. Some predisposing variants are common in different forms of cancer. These polymorphisms might be general risk factors for carcinogenesis.

Keywords: epidermal growth factor, cancer, polymorphism, signaling pathway 


\section{Introduction}

Representing $>1 \%$ of the whole allele frequency, single-nucleotide polymorphisms (SNPs) are the most frequent DNA variation. An SNP is the result of point mutations, it involves a single base pair and, usually, has no effect. The sequencing of the entire human genome was the start of a new research direction: genome-wide association studies (GWAS) [1, 2]. Their purpose is to identify a genetic susceptibility by testing the association with a disease for hundreds of thousands of SNPs in a GWAS. One of the many medical fields which can benefit from the identification of such genetic risk factors is oncology [3].

In 1985, Sporn and Roberts explained that malignant cells produce elevated levels of growth factors to maintain their proliferation rate. This observation was followed by an enormous number of research projects which confirmed the importance of growth factors for cancer pathogenesis and described the mechanisms and molecular interactions involved in this process [4].

Discovered in 1962, epidermal growth factor (EGF) is encoded by prepro-EGF gene, on chromosome 4 (4q25-q27) [5]. In 1986, Cohen and Levi-Montalcini won the Nobel Prize for Medicine for the description of the peptide structure of EGF [6]. It is the member of a family of growth factors which can bind to the same receptor: EGF receptor (EGFR, ErbB1). EGFR is a type I transmembrane protein with intrinsic tyrosine kinase activity. When a ligand binds to EGFR, the receptor forms homodimers (dimerization with an identical EGFR) or heterodimers with other members of its family. The dimerization activates the intrinsic tyrosine kinase and the autophosphorylation of the C-terminal tyrosine residue, facilitating the recruitment of signaling molecules. Signaling pathways involved in cell proliferation, differentiation, apoptosis, such as Ras/Raf/MEK/ERK, JAK/STAT, PI3K/AKT/mTOR, and PLC/PKC, are activated. A series of malignant tumors were associated with an amplification of EGF/EGFR pathway, caused by increased EGF synthesis, EGFR overexpression, and EGFR mutations. Progression of cancer and metastasis are correlated with the level of EGF/EGFR. Considering the role it plays in cancer pathogenesis, EGFR inhibition is used as a target in oncologic therapies [5]

Considering that the connection of EGF/EGFR signaling pathway with malignant tumors is well established [5], it is expected to identify the presence of predisposing SNPs in the encoding regions for various forms of cancer. In the era of GWASs, the quantity of information about susceptibility and genetic factors, such as SNPs, is abundant [3] and reviews are mandatory, as a way to organize all this knowledge and highlight the issues that can be addressed in the clinic. Therefore, the aim of this study was 
to analyze the data extracted from genomic studies regarding cancer and EGF/EGFR pathways and present it.

\section{Materials and Methods}

We conducted a search on PubMed, Scopus, Web of Science, and Science Direct in May 2020, using the following search keywords: EGF OR epidermal growth factor AND polymorphism AND cancer OR neoplasia OR tumor. After a thorough investigation of database interrogation results, we included in our study the most relevant 40 full-text articles published in the last 10 years.

\section{Results}

We identified relevant polymorphisms of the EGF signaling pathway that were involved in the development and progression of hepatocellular carcinoma (HCC), esophageal cancer, gastric cancer, colorectal cancer, glioma, lung cancer, breast cancer, cervical cancer, and head and neck cancer. The SNPs, their connection to these malignancies, and the studies that identified the relevant variants are presented below, starting with a presentation of each cancer and its predisposing polymorphisms and ending with the discussion of these findings.

\subsection{Epidermal Growth Factor (EGF) Signaling Pathway and Liver Cancer}

EGF was identified as a factor which induces malignant transformation among hepatocytes, and it has an important impact on the progression of tumor too, as a hepatocytic mitogen factor. A polymorphism of EGF gene, position 61 A $>G$, SNP rs4444903, results in an increased EGF production in liver [7].

Several studies investigated the risk of developing HCC and the presence of rs4444903 SNPs. An association between $A / G$ and $G / G$ genotypes and increased risk of HCC was identified [8-10].

Two case-control studies were conducted in parallel, one of them on non-Asian population from Los Angeles (region having one of the lowest incidences of HCC in the world), and the other on natives from Guangxi, China (population having one of the highest incidences of HCC in the world). Among the non-Asian Los Angeles population, there was a $78 \%$ increased risk of developing liver cancer in patients who 
had at least one $61^{*} \mathrm{G}$ allele (rs4444903 SNP), which is associated with high activity and EGF production, in comparison to EGF A/A genotype. The results were, however, not statistically significant. Among the Chinese population, no association was identified between this polymorphism and HCC risk [7].

Based on the findings resulted from the numerous case-control studies regarding rs4444903 SNP and HCC, various meta-analyses were conducted. We identified six meta-analyses that revealed an association between $\mathrm{G}$ allele and an elevated risk of developing HCC. The A allele is considered a protective factor [11-16].

\subsection{Epidermal Growth Factor (EGF) Signaling Pathway and Esophageal Cancer}

A genome-wide association study conducted on 1,942 patients with esophageal squamous cell carcinoma, 1,758 patients with gastric cancer, and 2,111 controls investigated 127 genes of the EGF pathway. GNAI3, CHRNE, PAK4, WASL, ITCH genes were associated with esophageal squamous cell carcinoma, but there was no significant association with the pathway [17].

\subsection{Epidermal Growth Factor (EGF) Signaling Pathway and Gastric Cancer}

The genome-wide association study investigating 127 genes of the EGF pathway also concluded that FYN, MAPK8, MAP2K4, GNAI3, MAP2K1, TLN1, PRLR, PLCG2, RPS6KB2, $P I K 3 R$ genes, and the EGFR pathway were associated with gastric cancer [17].

A case-control study conducted on high-risk Chinese population from Jiangxi investigated seven EGFR exon SNPs. The study identified only one variant, rs2072454, T allele and TT genotype, significantly associated with elevated risk of developing gastric cancer [18]. AG/GG rs2237051 (non-synonymous exon region) and AG/GG rs3733625 ( $3^{\prime}$ untranslated region) variants of EGF gene were identified as susceptibility factors in a study conducted on Chinese population [19]. A study based on Mexican population suggested the association of $-216 \mathrm{G}>\mathrm{T}, 191 \mathrm{C}>\mathrm{A}$ polymorphisms of EGFR and the risk of developing gastric cancer [20].

Rs4444903 was also investigated regarding gastric cancer [21]. A study conducted in Portugal on Caucasian subjects identified different risk profile associated with +61 $A / G$ polymorphism, depending on the patient's sex. Female carriers of $A$ variant had a low risk of developing gastric cancer. A possible explanation for this phenomenon is 
the lower secretion of gastric acid than men. Male carriers of A polymorphism had an elevated risk of developing this neoplasia [22]. A meta-analysis conducted in 2014 on six case-control studies strongly suggested that this polymorphism leads to elevated gastric cancer risk, especially in Asian population [21].

\subsection{Epidermal Growth Factor (EGF) Signaling Pathway and Col- orectal Cancer}

A study conducted on Iranian population investigated the association of rs2298979 polymorphism, located in a noncoding region - intron 1 of EGF gene, with colorectal cancer. $A / G$ genotype carriers were identified as less susceptible than the carriers of A/A genotype [23].

The association between colorectal cancer and SNP rs4444903 was also researched. A study based on Syrian subjects demonstrated that $A / A$ variant of R521K is a protective factor for colorectal cancer [24], while another study based on Chinese population indicated an association of EGF A61G polymorphism with elevated risk for developing colorectal cancer [25]. Two studies failed to highlight an association between $+61 \mathrm{~A}>\mathrm{G}$ polymorphism and elevated colorectal cancer risk [26, 27].

A meta-analysis based on nine studies concluded that EGF A61G polymorphism might be a risk factor for colorectal cancer development ( $G$ allele in the Caucasian group and GG genotype in both Caucasian and Asian populations) [28].

\subsection{Epidermal Growth Factor (EGF) Signaling Pathway and Glioma}

A higher risk of developing glioma was associated with -191 C/A SNP of EGFR. This polymorphism was also identified as a prognostic factor in patients with glioblastoma. Short $(C A) n$ repeats $(<17)$ were associated with elevated risk, especially for glioblastoma and oligodendroglioma [29].

A study conducted in 2012 on Han Chinese population identified two polymorphisms (rs1468727 and rs730437) in the EGFR gene which are associated with increased risk of glioma. AATT haplotype had a protective effect, while CGTC haplotype was associated with an increased risk of developing glioma. The findings of this study including Han Chinese subjects were not consistent with the results of previous studies on European populations, therefore ethnic differences are strongly suggested [30].

Numerous studies have identified an association between $+61 G$ allele of EGF and high risk for developing glioma. A study conducted on Chinese population from eastern 
China concluded that genotypes $+61 \mathrm{G} / \mathrm{A}$ and $+61 \mathrm{~A} / \mathrm{A}$ were associated with higher risk of glioma, in comparison to $\mathrm{G} / \mathrm{G}$, considered wild type [31]. A meta-analysis including nine studies concluded that EGF $+61 \mathrm{G} / \mathrm{A}$ polymorphism is a risk factor for glioma [32]. A meta-analysis including six case-control studies concluded that this polymorphism is a risk factor for European population, but a protective factor regarding Chinese population [33]. Another meta-analysis based on seven studies concluded that EGF $+61 \mathrm{G} / \mathrm{A}$ polymorphism is associated with a higher risk of developing glioma in Asian population, but not in Caucasians [34].

\subsection{Epidermal Growth Factor (EGF) Signaling Pathway and Breast Cancer}

A study investigating EGFR SNPs and breast cancer risk identified associations among SNPS in EGFR gene and the risk for development of breast cancer. After multiple comparisons, none of the results remained statistically significant [35].

Rs11543848 (EGFR R497K) and rs1136201 (HER2 1655V) were associated with the risk of developing breast cancer [36]. Rs1136201 (Ile 655 Val) genetic polymorphism of human epidermal growth factor receptor-2 (HER2) was identified as a possible risk factor for developing breast cancer in Brazilian population [37].

\subsection{Epidermal Growth Factor (EGF) Signaling Pathway and Lung Cancer}

A study based on Jordan population identified rs2233947 EGFR polymorphism as a risk factor for lung cancer development. This SNP is located in the exon 25 and does not produce an amino acid modification. The A allele is protective for the development of lung cancer [38].

Rs712829 EGFR polymorphism (GG+GT), rs2072454, -191C/A (rs712830), rs6965469, rs763317, and TCCG haplotype of rs712829, rs712830, rs2072454, rs11543848 were associated with lung cancer [39-41].

A study conducted on Indian population identified +61 AG and GG genotypes as risk factors for developing non-small cell lung adenocarcinoma [42], while a recently published meta-analysis based on six case-control studies failed to identify an association between 61A/G EGF polymorphism and the development of lung cancer [43]. 


\subsection{Epidermal Growth Factor Receptor (EGFR) Family and Osteosarcoma}

Rs1136201 and rs1058808 polymorphisms of HER2 (ErbB2) and CTGG haplotype were associated with an elevated risk of developing osteosarcoma in Han Chinese population [44].

\subsection{Epidermal Growth Factor (EGF) Signaling Pathway and Cervi- cal Cancer}

A study investigating SNPs in members of ERBB family (EGFR, ERBB2, ERBB3, ERBB4) identified 14 polymorphisms that were significantly over transmitted (from parents to their offspring) in women who developed cervical cancer. Four SNPs were inside intron 1 of EGFR and two other were located in intron 24 of ERBB4. The SNPs identified in EGFR were in the proximity of enhancers, silencers, and important functional regions [45].

\subsection{Epidermal Growth Factor (EGF) Signaling Pathway and Head and Neck Squamous Cell Carcinoma}

Rs12535536, rs2075110, rs1253871, rs845561, rs6970262 SNPs located inside EGFR introns and synonymous rs2072454 EGFR SNP were significantly associated with elevated risk of developing head and neck squamous cell carcinoma, while rs12538371, rs845561, rs6970262 were associated with elevated risk among patients who never used tobacco [46].

\section{Discussion}

We identified numerous studies investigating the presence of single nucleotide polymorphisms of genes encoding proteins involved in EGF signaling pathway and the risk of developing different forms of malignant tumors. Some of these polymorphisms, such as rs 4444903 , were widely studied and meta-analyses were conducted to increase the strength of the results. The findings can be explained by the functional importance of these SNPs, many of them leading to an increase in the pathway activity, and, in the end, promoting the progression of the malignancy $[21,39,40]$. Genetic variants of the EGF signaling pathway and EGFR family were identified as risk factors for various tumors, such as: HCC [8-16], esophageal cancer [17], gastric cancer [17-22], colorectal cancer 
[23-28], glioma [29-34], lung cancer [38-42], breast cancer [36, 37], cervical cancer [45], and head and neck cancer [46]. EGF is important for tumor survival, progression, metastasis, and angiogenesis [19]. EGF signaling pathway might be relevant not only for the risk of developing different forms of cancer, but also for prognosis. For example, a study conducted on Indian population also identified an association between +61AG and GG genotypes and overall reduced survival in lung cancer patients [42].

The finding of the studies investigating the same SNPs and the development of a particular form of cancer were different. Some studies suggest an association between a malignant tumor and these variants, while others fail to identify a statistically significant result $[26,27,43]$. We identified numerous sources that might lead to inconsistent results: the sample size - from tens of subjects to thousand, study population and its different genetic and specific environmental risk factors that can modify susceptibility, data analysis tools and thresholds for significance, and inclusion and exclusion criteria for study population. However, most studies had similar conclusions regarding the most relevant polymorphisms.

One of the most relevant polymorphisms, according to the studies we identified, is rs4444903 SNP. This polymorphism is located in $5^{\circ}-$ untranslated region of EGF gene and $+61 \mathrm{GG}$ and $+61 \mathrm{AG}$ variants lead to an increased protein expression compared to +61 AA genotype [21]. This polymorphism has been widely studied regarding various forms of cancer and an association of the risk of developing tumors has been identified by most studies: liver cancer [8-16], gastric cancer [21, 22], colorectal cancer [25], glioma [31-34], and lung cancer [42]. Few studies failed to identify this association [26, 27, 43]. The results from case-control studies were different, depending on study population. It was suggested that rs4444903 SNP is a low-penetrance susceptibility biomarker [33].

Considering the functional relevance and the known association with an increased activity of EGFR signaling pathway of this variant, the positive results of the studies are supported by evidence provided by fundamental studies [21]. AA genotype was identified as a protective factor $[12,15]$, which is consistent with its functional importance: the protein production is not very high, while $A G$ and $G G$ genotypes were suggested to be risk factors, which is explained by the higher production of the protein and an increased activity of the pathway, leading in the end to an increased proliferation and survival of the malignant cell [8-16, 21, 22, 25, 31-34, 42].

The promoter region is essential to produce protein and polymorphisms of this sequence might lead to important functional alterations. $-216 \mathrm{G} / \mathrm{T}$ and $-191 \mathrm{C} / \mathrm{A}$ SNPs, both located inside the promoter region of EGFR gene, and polymorphic (CA)n 
microsatellite sequence in the first intron have regulatory functions regarding transcription [29]. -191C/A SNP results in an increased production of EGFR and it occurs at the binding site of the promoter region with a transcription factor $[39,40]$. While -216 G/T genotype was not significantly associated with glioma, -191 C/A SNP was associated with higher risk of developing glioma. Also, short $(C A) n$ repeats $(<17)$ were associated with elevated risk, especially for glioblastoma and oligodendroglioma [29]. Both $-216 G>T$ and $191 \mathrm{C}>\mathrm{A}$ were associated with the risk of developing gastric cancer [20]. -191C/A (rs712830) was also associated with lung cancer [39, 40]. Considering the location inside EGFR gene, functional importance and the results of previous studies, these polymorphisms and association with cancer should be further investigated.

EGF Receptor or erB family members are HER1 (EGFR, erbB1), HER2 (erbB2, neu), HER3 (erbB3), and HER4 (erbB4) [36]. Polymorphisms of HER2 were also identified as risk factors in both breast cancer and osteosarcoma [37, 44]. Overall, ErB family might be relevant for malignancy pathogenesis and further studies should be conducted to determine its importance for cancer development.

To the best of our knowledge, this narrative review is the first article that synthesizes and analyses the information regarding different types of tumors and polymorphisms as risk factors. All studies we identified were based on a specific tumor and associated polymorphisms. We consider it is important to summarize all this information together, because, as mentioned earlier, some SNPs are relevant for various forms of malignancy and polymorphisms of EGF signaling pathway might be relevant for cancer pathogenesis in general, not only for a specific malignant cell and it can be important to identify such variants for most tumors.

The results of our study were affected by several factors: limited access to research conducted in this area and the studies we included had wide differences regarding sample size, study population, environmental risk factors.

Genetic and molecular screening can have a major role in improving the diagnostic efficiency and treatment response in patients with cancer [47]. Identifying genetic variants of EGF signaling pathway as risk factors is important to determine if a subject is at risk of developing a malignancy and to use this information to take preventive steps and to start periodic screening. Furthermore, the proteins involved in this cellular pathway can be used as future targets in patients who have an increased activity of EGF signaling.

Future research is required to investigate the association between EGF signaling pathway and malignancies. These studies need larger sample size, and they should 
take into consideration sex differences together with the genetic and environmental factors of the study population.

\section{Conclusion}

Our narrative review confirms the presence of predisposing polymorphisms of the genes related to EGF signaling pathway, which are relevant for the development of various forms of cancer, such as HCC, esophageal cancer, gastric cancer, colorectal cancer, glioma, lung cancer, breast cancer, cervical cancer, and head and neck cancer. Rs4444903 EGF gene variants have been widely studied and the association with different tumors has been confirmed by numerous meta-analyses. Functionally important SNPs of EGFR gene are -191C/A and -216G > T. Other relevant SNPs might be identified by studies on larger samples and further investigation should be conducted to use these variants as risk evaluation and prognosis tools or as therapeutic targets.

\section{Acknowledgements}

None

\section{Ethical Considerations}

Not applicable.

\section{Competing Interests}

None.

\section{Availability of Data and Material}

None. The article is a review.

\section{Funding}

None. 


\section{References}

[1] Shen, W., Tian, Y., Ran, T., et al. (2015). Genotyping and quantification techniques for single-nucleotide polymorphisms. Trends in Analytical Chemistry, vol. 69, pp. 1-13. Retrieved from: https://linkinghub.elsevier.com/retrieve/pii/S0165993615000953

[2] Hanchard, N. A. (2005). Genetic susceptibility and single-nucleotide polymorphisms. Seminars in Fetal \& Neonatal Medicine, vol. 10, no. 3, pp. 283-289. Retrieved from: https://linkinghub.elsevier.com/retrieve/pii/S1744165X05000090

[3] Fareed, M. and Afzal, M. (2013). Single nucleotide polymorphism in genome-wide association of human population: a tool for broad spectrum service. Egyptian Journal of Medical Human Genetics, vol. 14, no. 2, pp. 123-134. Retrieved from: https:// linkinghub.elsevier.com/retrieve/pii/S1110863012000687

[4] Normanno, N., De Luca, A., Bianco, C., et al. (2006). Epidermal growth factor receptor (EGFR) signaling in cancer. Gene, vol. 366, no. 1, pp. 2-16. Retrieved from: https: //linkinghub.elsevier.com/retrieve/pii/S0378111905006347

[5] Zeng, F. and Harris, R. C. (2014). Epidermal growth factor, from gene organization to bedside. Seminars in Cell and Developmental Biology, vol. 28, pp. 2-11. Retrieved from: https://linkinghub.elsevier.com/retrieve/pii/S1084952114000135

[6] Esquirol Caussa, J. and Herrero Vila, E. (2015). Epidermal growth factor, innovation and safety. Med Clínica, vol. 145, no. 7, pp. 305-312. Retrived from: https://linkinghub. elsevier.com/retrieve/pii/S2387020616000930

[7] Yuan, J. M., Fan, Y., Ognjanovic, S., et al. (2013). Genetic polymorphisms of epidermal growth factor in relation to risk of hepatocellular carcinoma: two case-control studies. BMC Gastroenterology, vol. 13, no. 1, p. 32. Retrieved from: https://bmcgastroenterol. biomedcentral.com/articles/10.1186/1471-230X-13-32

[8] Suenaga, M., Yamada, S., Fujii, T., et al. (2013). A functional polymorphism in the epidermal growth factor gene predicts hepatocellular carcinoma risk in Japanese hepatitis C patients. OncoTargets and Therapy, vol. 6, pp. 18051812. Retrieved from: http://www.dovepress.com/a-functional-polymorphism-in-theepidermal-growth-factor-gene-predicts-peer-reviewed-article-OTT

[9] Abu Dayyeh, B. K., Yang, M., Fuchs, B. C., et al. (2011). A functional polymorphism in the epidermal growth factor gene is associated with risk for hepatocellular carcinoma. Gastroenterology, vol. 141, no. 1, pp. 141-149. Retrieved from: https: //linkinghub.elsevier.com/retrieve/pii/S0016508511004161

[10] Shi, H. Z., Ren, P., Lu, Q. J., et al. (2012). Association between EGF, TGF- $\beta 1$ and TNF- $\alpha$ gene polymorphisms and hepatocellular carcinoma. Asian Pacific Journal of Cancer 
Prevention, vol. 13, no. 12, pp. 6217-6220. Retrieved from: http://koreascience.or.kr/ journal/view.jsp?kj=POCPA9\&py=2012\&vnc=v13n12\&sp=6217

[11] Jiang, G., Yu, K., Shao, L., et al. (2015). Association between epidermal growth factor gene $+61 \mathrm{~A} / \mathrm{G}$ polymorphism and the risk of hepatocellular carcinoma: a metaanalysis based on 16 studies. BMC Cancer, vol. 15, no. 1, p. 314. Retrieved from: http://bmccancer.biomedcentral.com/articles/10.1186/s12885-015-1318-6

[12] Zhong, J. H., You, X. M., Gong, W. F., et al. (2012). Epidermal growth factor gene polymorphism and risk of hepatocellular carcinoma: a meta-analysis. PLOS ONE, vol. 7, no. 3, p. e32159. Retrieved from: https://dx.plos.org/10.1371/journal.pone.0032159

[13] Yang, Z., Wu, Q., Shi, Y., et al. (2012). Epidermal growth factor 61A>G polymorphism is associated with risk of hepatocellular carcinoma: a meta-analysis. Genetic Testing and Molecular Biomarkers, vol. 16, no. 9, pp. 1086-1091. Retrieved from: http://www. liebertpub.com/doi/10.1089/gtmb.2012.0050

[14] Li, Y. L., Tian, Z., Zhao, L., et al. (2014). Association between the EGF rs4444903 polymorphism and liver cancer susceptibility: a meta-analysis and meta-regression. Genetics and Molecular Research, vol. 13, no. 4, pp. 8066-8079. Retrieved from: http://www.funpecrp.com.br/gmr/year2014/vol13-4/pdf/gmr3575.pdf

[15] Zhang, Y., Zhang, J., Shen, N., et al. (2016). EGF rs4444903a allele may decrease hepatocellular carcinoma risk in chinese individuals: a meta-analysis. International Journal of Clinical and Experimental Medicine, vol. 9, no. 3, pp. 5719-5727. Retrieved from: http://www.ijcem.com/files/ijcem0009615.pdf

[16] Sun, S., Jin, G. J., Zhao, Y., et al. (2015). Association between the epidermal growth factor $61^{*} \mathrm{~A} / \mathrm{G}$ polymorphism and hepatocellular carcinoma risk: a metaanalysis. Asian Pacific Journal of Cancer Prevention, vol. 16, no. 7, pp. 30093014. Retrieved from: http://koreascience.or.kr/journal/view.jsp?kj=POCPA9\&py= $2015 \& \mathrm{vnc}=\mathrm{v} 16 \mathrm{n} 7 \& \mathrm{sp}=3009$

[17] Li, W. Q., Hu, N., Wang, Z., et al. (2013). Genetic variants in epidermal growth factor receptor pathway genes and risk of esophageal squamous cell carcinoma and gastric cancer in a Chinese population. PLoS One, vol. 8, no. 7, e68999. Retrieved from: http://www.ncbi.nlm.nih.gov/pubmed/23874846

[18] Zhang, J., Zhan, Z., Wu, J., et al. (2013). Association among polymorphisms in EGFR gene exons, lifestyle and risk of gastric cancer with gender differences in Chinese Han subjects. PLoS ONE, vol. 8, no. 3, e59254. Retrieved from: https://dx.plos.org/ 10.1371/journal.pone.0059254 
[19] Zhan, Z., Chen, Y., Wu, J., et al. (2013). Functional epidermal growth factor gene polymorphisms and risk of gastric cancer. Oncology Letters, vol. 5, no. 2, pp. 631636. Retrived from: https://www.spandidos-publications.com/10.3892/ol.2012.1041

[20] Torres-Jasso, J. H., Marín, M. E., Luna Santiago, E. S., et al. (2015). EGFR gene polymorphisms -216G $>$ T and -191C $>A$ are risk markers for gastric cancer in Mexican population. Genetics and Molecular Research, vol. 14, no. 1, pp. 1802-1807. Retrieved from: http://www.funpecrp.com.br/gmr/year2015/vol14-1/pdf/gmr4537.pdf

[21] Peng, Q., Li, S., Qin, X., et al. (2014). EGF +61A/G polymorphism contributes to increased gastric cancer risk: evidence from a meta-analysis. Cancer Cell International, vol. 14, no. 1, p. 134. Retrieved from: http://cancerci.biomedcentral. com/articles/10.1186/s12935-014-0134-4

[22] Araújo, A. P., Costa, B. M., Pinto-Correia, A. L., et al. (2011). Association between EGF $+61 \mathrm{~A} / \mathrm{G}$ polymorphism and gastric cancer in caucasians. World Journal of Gastroenterology, vol. 17, no. 4, pp. 488-492. Retrieved from: https://www.ncbi.nlm. nih.gov/pmc/articles/PMC3027015/

[23] Chaleshi, V., Haghighi, M. M., Savabkar, S., et al. (2013). Correlation between the EGF gene intronic polymorphism, rs2298979, and colorectal cancer. Oncology Letters, vol. 6, no. 4, pp. 1079-1083. Retrieved from: https://www.spandidos-publications. com/10.3892/ol.2013.1481

[24] Mustafa, O. H., Hamzeh, A. R., Ghabreau, L., et al. (2013). Allele frequencies of the epidermal growth factor receptors polymorphism R521K in colorectal cancer patients and healthy subjects indicate a risk-reducing effect of K521 in Syrian population. North American Journal of Medical Sciences, vol. 5, no. 3, pp. 202-206. Retrieved from: http://www.najms.org/text.asp?2013/5/3/202/109189

[25] Zhu, X., Shen, Y., and Xie, Q. (2019). The association between EGF A61G polymorphism and risk of colorectal cancer in a Chinese population: a casecontrol study. Bioscience Reports, vol. 39, no. 5, BSR20190495. Retrieved from: https://portlandpress.com/bioscirep/article/doi/10.1042/BSR20190495/219161/ The-association-between-EGF-A61G-polymorphism-and

[26] Lau, T. P., Roslani, A. C., Lian, L. H., et al. (2014). Association between EGF and VEGF functional polymorphisms and sporadic colorectal cancer in the Malaysian population. Genetics and Molecular Research, vol. 13, no. 3, pp. 5555-5561. Retrieved from: http://www.funpecrp.com.br/gmr/year2014/vol13-3/pdf/gmr3635.pdf

[27] Chaleshi, V., Haghighi, M. M., Javadi, G. R., et al. (2013). The effect of 5'untranslated region polymorphism in EGF gene, rs4444903, on colorectal cancer. 
Gastroenterology and Hepatology From Bed to Bench, vol. 6, no. 3, pp. 129-135. Retrieved from: http://www.ncbi.nlm.nih.gov/pubmed/24834259

[28] Zhu, Y., Chen, Z. H., Jiang, H. G., et al. (2019). The genetic association between EGF A61G polymorphism (rs4444903) and risk of colorectal cancer: an update metaanalysis and trial sequential analysis. Medicine, vol. 98, no. 2, e14007. Retrieved from: http://journals.Iww.com/00005792-201901110-00041

[29] Costa, B. M., Viana-Pereira, M., Fernandes, R., et al. (2011). Impact of EGFR genetic variants on glioma risk and patient outcome. Cancer Epidemiology, Biomarkers \& Prevention, vol. 20, no. 12, pp. 2610-2617. Retrieved from: http://www.ncbi.nlm.nih. gov/pubmed/21960689

[30] Hou, W. G., Ai, W. B., Bai, X. G., et al. (2012). Genetic variation in the EGFR gene and the risk of glioma in a Chinese Han population. PLoS ONE, vol. 7, no. 5, e37531. Retrieved from: https://dx.plos.org/10.1371/journal.pone.0037531

[31] Wang, S., Zhao, Y., Ruan, Z., et al. (2010). Association between EGF +61 G/A and glioma risk in a Chinese population. BMC Cancer, vol. 10, p. 221. Retrieved from: http://bmccancer.biomedcentral.com/articles/10.1186/1471-2407-10-221

[32] Chen, X., Yang, G., Zhang, D., et al. (2014). Association between the epidermal growth factor $+61 \mathrm{G} / \mathrm{A}$ polymorphism and glioma risk: a meta-analysis. PLOS ONE, vol. 9, no. 4, e95139. Retrieved from: https://dx.plos.org/10.1371/journal.pone.0095139

[33] Tan, D., Xu, J., Li, Y., et al. (2010). Association between +61G polymorphism of the EGF gene and glioma risk in different ethnicities: a meta-analysis. Tohoku Journal of Experimental Medicine, vol. 222, no. 4, pp. 229-235. Retrieved from: http://joi.jlc. jst.go.jp/JST.JSTAGE/tjem/222.229?from=CrossRef

[34] Xu, X., Xi, L., Zeng, J., et al. (2012). A functional +61G/A polymorphism in epidermal growth factor is associated with Glioma risk among asians. PLOS ONE, vol. 7, no. 7, e41470. Retrieved from: https://dx.plos.org/10.1371/journal.pone.0041470

[35] Connor, A. E., Baumgartner, R. N., Baumgartner, K. B., et al. (2013). Epidermal Growth Factor Receptor (EGFR) polymorphisms and breast cancer among Hispanic and non-Hispanic White women: The Breast Cancer Health Disparities Study. International Journal of Molecular Epidemiology and Genetics, vol. 4, no. 4, pp. 235-249. Retrieved from: http://www.pubmedcentral.nih.gov/articlerender. fcgi?artid=PMC3852643

[36] Abdraboh, N. R., Shehata, H. H., Ahmed, M. B., et al. (2013). HER1 R497K and HER2 $1655 \mathrm{~V}$ polymorphisms are linked to development of breast cancer. Disease Markers, vol. 34, no. 6, pp. 407-17. Retrieved from: https://www.ncbi.nlm.nih.gov/pmc/articles/ PMC3810125/ 
[37] de Almeida, F. C., Banin Hirata, B. K., Ariza, C. B., et al. (2018). HER2 lle655Val polymorphism is negatively associated with breast cancer susceptibility. Journal of Clinical Laboratory Analysis, vol. 1, no. 6, e22406. Retrieved from: http://doi.wiley. com/10.1002/jcla.22406

[38] Bashir, N. A., Morad, F. R., Khabour, O. F., et al. (2019). The EGFR rs2233947 polymorphism is associated with lung cancer risk: a study from Jordan. Acta Biochimica Polonica, vol. 66, no. 3, pp. 315-319. Retrieved from: http://www.ncbi. nlm.nih.gov/pubmed/31518089

[39] Bashir, N. A., Ragab, E. S., Khabour, O. F., et al. (2018). The association between epidermal growth factor receptor (EGFR) gene polymorphisms and lung cancer risk. Biomolecules, vol. 8, no. 3, p. 53. Retrieved from: http://www.mdpi.com/2218-273X/ $8 / 3 / 53$

[40] Beg, M. M. A., Fahdil, S. R., Yadav, P., et al. (2019). Association of EGFR 1 gene alteration and their association with lung adenocarcinoma patients. Asian Pacific Journal of Cancer Prevention, vol. 20, no. 3, pp. 825-830. Retrieved from: http: //journal.waocp.org/article_82575.html

[41] Liu, C., Xu, X., and Zhou, Y. (2015). Association between EGFR polymorphisms and the risk of lung cancer. International Journal of Clinical and Experimental Pathology, vol. 8, no. 11, pp. 15245-15249. Retrieved from: http://www.ncbi.nlm.nih.gov/pubmed/ 26823874

[42] Masroor, M., Amit, J., Javid, J., et al. (2015). Clinical implication of EGF A61G polymorphism in the risk of non small cell lung adenocarcinoma patients: a case control study. Asian Pacific Journal of Cancer Prevention, vol. 16, no. 17, pp. 7529 7534. Retrieved from: http://koreascience.or.kr/journal/view.jsp?kj=POCPA9\&py= $2015 \& \mathrm{vnc}=\mathrm{v} 16 \mathrm{n} 17 \& \mathrm{sp}=7529$

[43] Chen, Q., Zheng, Y., Wu, B., et al. (2020). Association between polymorphisms of epidermal growth factor 61 and susceptibility of lung cancer. Medicine, vol. 99, no. 17, e19456. Retrieved from: https://journals.Iww.com/10.1097/MD.0000000000019456

[44] Xin, D. J., Shen, G. D., and Song, J. (2015). Single nucleotide polymorphisms of HER2 related to osteosarcoma susceptibility. International Journal of Clinical and Experimental Pathology, vol. 8, no. 8, pp. 9494-9499. Retrieved from: http://www. ncbi.nlm.nih.gov/pubmed/26464710

[45] Ma, D., Hovey, R. L., Zhang, Z., et al. (2013). Genetic variations in EGFR and ERBB4 increase susceptibility to cervical cancer. Gynecologic Oncology, vol. 131, no. 2, pp. 445-450. Retrieved from: https://linkinghub.elsevier.com/retrieve/pii/ S0090825813010780 
[46] Fung, C., Zhou, P., Joyce, S., et al. (2015). Identification of epidermal growth factor receptor (EGFR) genetic variants that modify risk for head and neck squamous cell carcinoma. Cancer Letters, vol. 357, no. 2, pp. 549-556. Retrieved from: https:// linkinghub.elsevier.com/retrieve/pii/S0304383514007514

[47] Bolocan, A., Stoian, R. V., Ion, D., et al. (2012). Peculiarities of diagnosis and treatment in the polyp-cancer sequence. Chirurgia, vol. 107, no. 1, pp. 66-70. Retrieved from: http://www.ncbi.nlm.nih.gov/pubmed/22480119 